\title{
Bem-Estar Subjetivo de Longevos Institucionalizados e Não Institucionalizados por meio do Pfister
}

\author{
Cristina Ribas Teixeira, Silvana Alba Scortegagna ${ }^{1}$ \\ Universidade de Passo Fundo, Passo Fundo-RS, Brasil \\ Sonia Regina Pasian \\ Universidade de São Paulo, Ribeirão Preto-SP, Brasil \\ Marilene Rodrigues Portella \\ Universidade de Passo Fundo, Passo Fundo-RS, Brasil
}

\section{RESUMO}

Este estudo investigou o Bem-Estar Subjetivo (BES) de longevos institucionalizados e não institucionalizados por meio do Teste das Pirâmides Coloridas de Pfister (TPC). Participaram 70 idosos com 80 anos e mais, 35 procedentes de instituições de longa permanência (GI) e 35 provenientes da comunidade (GNI), individualmente avaliados pelos seguintes instrumentos: questionário sociodemográfico, TPC, Escala de Afetos Positivos e Afetos Negativos, Escala de Depressão Geriátrica Abreviada e Escala de Autoestima de Rosenberg. Não houve diferenças estatisticamente significativas entre os grupos no TPC, mas sinalizaram preservação da afetividade, embora com funcionamento cognitivo menos elaborado (predomínio de tapetes). GI apresentou sinais de piores condições funcionais e afeto positivo estatisticamente menor (PANAS), com tendência a pior autoestima (EAR). Ambos os grupos evidenciaram sinais de vivências depressivas (GDS-15). Os achados contribuem para compreensão da psicodinâmica na velhice e futuro planejamento de estratégias de intervenções que fomentem o BES dos longevos.

Palavras-chave: métodos projetivos; bem-estar subjetivo; personalidade; envelhecimento, longevidade.

ABSTRACT - Subjective Well-being of Institutionalized and Non-Institutionalized Older Adults Using Pfister's Test This study investigated the subjective well-being (SWB) of institutionalized and non-institutionalized older adults using Pfister's Colors Pyramid Test (CPT). Participants were 70 older adults aged 80 years or over, with 35 being residents of Long Term Care Institutions (IG) and 35 living in the Community (CG). They were individually assessed through the application of a sociodemographic questionnaire, the CPT, the Positive and Negative Affections Scale, the Geriatric Depression Scale - short form, and the Rosenberg Self-esteem Scale. There were no statistically significant differences between the groups in the CPT, and they both presented preservation of affect, even with less elaborate cognitive functioning (predominance of carpets). The IG presented signs of worse functional conditions and statistically lower positive affection (PANAS), with a tendency for lower self-esteem (RSS). Both groups showed signs of depressive experiences (GDS-SF). The findings contribute to an understanding of psychodynamics in aging and to the future planning of intervention strategies that encourage SWB in older adults.

Keywords: projective methods; subjective well-being; personality; ageing; longevity.

RESUMEN - Bienestar Subjetivo de Ancianos Institucionalizados y no Institucionalizados por meio del Pfister

Este estudio investigó el Bienestar Subjetivo (BS) de los ancianos institucionalizados y no institucionalizados, por medio del Test de Pirámides de Colores de Pfister (TPC). Participaron 70 ancianos a partir de los 80 años, 35 de ellos procedentes de instituciones de larga permanencia (GI) y 35 provenientes de la comunidad (GNI), todos fueron individualmente evaluados de acuerdo con los siguientes instrumentos: cuestionario sociodemográfico, el TPC, las Escala de Afectos Positivos y Afectos Negativos, de Depresión Geriátrica Abreviada, y de Autoestima de Rosenberg. No hubo diferencias estadísticamente significativas entre los grupos en el TPC, si bien que señalaron preservación de la afectividad, pero con funcionamiento cognitivo menos elaborado (predominio de alfombras). El GI presentó signos de peores condiciones funcionales y afecto positivo estadísticamente menor (PANAS), con tendencia a peor autoestima (EAR). Ambos grupos evidenciaron signos de vivencias depresivas (GDS-15). Los hallazgos contribuyen a la comprensión de la psicodinámica en la vejez y futura planificación de estrategias de intervenciones que fomenten el BES de los ancianos.

Palabras clave: métodos proyectivos; bienestar subjetivo; personalidad; envejecimiento; longevidad.

O processo de envelhecimento emerge nos últimos tempos como um fenômeno carregado de significações.
Os baixos índices de mortalidade e os avanços das conquistas tecnológicas acenam para um aumento da 
população de indivíduos com 80 anos e mais (Carmo, Rangel, Ribeiro, \& Araújo, 2012). A certeza desse crescimento populacional e a maior sobrevivência de pessoas com redução da capacidade cognitiva, física, mental, despertam preocupações e incertezas, especialmente em relação ao bem-estar (Oliveira, Queiroz, \& Costa, 2012; Vicente et al., 2014) e as condições de moradia.

A busca pelas Instituições de Longa Permanência para Idosos (ILPIs), para os que possuem idade superior a 70 anos, está relacionada a déficits físicos e cognitivos, carência de cuidadores disponíveis e a conflitos familiares (Marinho, Vieira, Costa, \& Andrade, 2013). No geral, idosos residentes em ILPIs apresentam alto nível de dependência, dificuldades na realização das atividades diárias (Carmo et al., 2012; Marinho et al., 2013), elevado nível de afeto negativo e rebaixamento no afeto positivo (Hammarstrom \& Torres, 2012), sentimentos de solidão e dificuldade de interagir com outras pessoas (Dutra \& Silva, 2014) e sintomas depressivos (Vicente et al., 2014).

No contexto brasileiro, Carmo et al. (2012) investigaram os sentimentos dos idosos em ILPIs. Entre os resultados, a fragilidade que leva a dependência de outros, sentimentos de tristeza e de abandono foram fatores associados ao decréscimo do bem-estar subjetivo (BES) (Carmo et al., 2012; Dutra \& Silva, 2014; Hammarstrom \& Torres, 2012; Marinho et al., 2013).

O BES refere-se ao julgamento pessoal do quanto as pessoas são felizes, abrangendo aspectos emocionais (afeto positivo e negativo) e cognitivos (satisfação com a vida) (Hutz, Midgett, Pacico, Bastianello, \& Zanon, 2014; Zanon, Bastianello, Pacico, \& Hutz, 2013). O componente emocional está associado à intensidade e frequência com que as pessoas experimentam emoções positivas e negativas, como entusiasmo, alegria, raiva e tristeza (Dutra \& Silva, 2014; Hutz et al., 2014; Zanon et al., 2013). Experiências de eventos prazerosos tendem a se associar a afetos positivos, enquanto eventos de desprazer se vinculam a afetos negativos. No entanto, para se obter satisfação e felicidade, é necessário o acúmulo de experiências felizes (Oliveira et al., 2012; Zanon et al., 2013).

O componente cognitivo do BES reflete a percepção que o indivíduo tem da satisfação com sua própria vida (Hutz et al., 2014; Noronha, Martins, Campos, \& Mansão, 2015; Zanon et al., 2013). É considerado um indicador de saúde mental, componente essencial para a adaptação na velhice, pois quanto mais felizes as pessoas se sentem, maior a propensão a se considerarem saudáveis e de viverem mais (Diener \& Chan, 2011; Oliveira et al., 2012). Tinoco, Tinoco e Carvalho (2015) avaliaram 30 idosos, com idade entre 61 e 89 anos $(M=69,50$; $D P=6,58)$, residentes em seus lares, e constataram associação positiva entre o envelhecer saudável e variáveis cognitivas e psicossociais, vinculadas às condições de moradia e ao estar afetivamente acompanhado.

Ao analisar o campo de investigações sobre o BES, observa-se o predomínio do uso de escalas, com amostras jovens, o que denota a necessidade de investigações com o uso de ferramentas que possam trazer informações adicionais e abranger adultos mais velhos. Além das escalas, entre os instrumentos disponíveis para avaliação da dinâmica afetiva e cognitiva, destaca-se o Teste das Pirâmides Coloridas de Pfister (TPC), método projetivo não verbal e com características lúdicas (Franco \& Villemor-Amaral, 2012; Villemor-Amaral, 2013) que avalia componentes funcionais da personalidade. No Brasil, várias pesquisas apontaram evidências de sua validade clínica, incluindo diferentes contextos psicopatológicos: transtorno do pânico (Villemor-Amaral, Farah, \& Primi, 2004), esquizofrenia (Villemor-Amaral, Primi, Franco, Farah, \& Cardoso, 2005), na psicodinâmica dos transtornos alimentares (Oliveira-Cardoso \& Santos, 2014), na depressão (Nogueira, 2013; Villemor-Amaral et al., 2004).

A literatura científica também versa sobre a utilização do TPC em diferentes segmentos etários, incluindo crianças (Villemor-Amaral, Biasi, Cardoso, Pavan, \& Tavella, 2015; Villemor-Amaral, Tavella, Cardoso, Biasi, \& Pavan, 2014), adolescentes e adultos (Nogueira, 2013; Oliveira-Cardoso \& Santos, 2014; Pasian, Barroso, \& Theodozio, 2014; Villemor-Amaral et al., 2004; VillemorAmaral et al., 2015) e idosos (Bastos-Formighieri \& Pasian, 2012; Oliveira, Pasian, \& Jacquemin, 2001). Diante do conjunto desses achados empíricos, o método de Pfister se mostra como recurso promissor na investigação psíquica de diferentes etapas do desenvolvimento, em diversos contextos socioculturais e clínicos. Pode, portanto, funcionar como rico instrumento na investigação de processos adaptativos e socioafetivos vinculados à institucionalização e ao BES, sobretudo da crescente população de idosos no Brasil e no mundo.

Apesar dessas possibilidades, a temática de idosos residentes em ILPIs e suas características de funcionamento socioafetivo ainda foram pouco investigadas no Brasil. Identificou-se apenas um estudo empírico a respeito, desenvolvido por Oliveira et al. (2001). Os autores buscaram comparar a vivência afetiva de grupo de idosas residentes em ILPIs com idosas da comunidade, utilizando o TPC como recurso investigativo. Os resultados mostraram maior porcentagem do aspecto formal "estruturas" no grupo de idosas não institucionalizadas e o predomínio de "tapetes" em idosas institucionalizadas, apontando indicadores de funcionamento lógico menos elaborado e com menor abstração nesse último grupo de mulheres. Em ambos os grupos, com relação às escolhas cromáticas, evidenciou-se sinais de preservação da vitalidade afetiva nas idosas, contrariando estereótipos de obrigatório decréscimo psíquico com o envelhecimento.

Em estudo posterior, Bastos-Formighieri e Pasian (2012) buscaram investigar características do funcionamento afetivo em idosos não pacientes. Avaliaram, desse modo, 100 indivíduos de 60 a 75 anos de idade, com diferentes níveis de escolaridade, e estabeleceram padrões normativos do TPC para essa faixa etária no 
contexto do Brasil, ainda que preliminares. No geral, os idosos apresentaram diferenças estatisticamente significativas na distribuição de frequências de escolhas em sete, das 10 cores do instrumento, quando comparados a amostra normativa de adultos não pacientes de Villemor-Amaral (2005). A preservação da dinâmica afetiva, sinais de adequada receptividade aos estímulos e presença de distintos arranjos defensivos dos idosos foram destacados, apontando especificidades na dinâmica psíquica dessa faixa etária, a ser aprofundada em futuras investigações. As cores verde (vd), vermelho (vm) e o azul (az) foram as mais utilizadas pelas idosas, ratificando indicadores de preservação da vitalidade afetiva dessa fase do desenvolvimento, averiguada em estudo prévio (Oliveira et al., 2001).

Nota-se, portanto, que a velhice, na contemporaneidade, é uma etapa da vida que merece novos investimentos de pesquisa, tendo em vista os desafios que a realidade impõe. Aliada a maior sobrevivência de pessoas com natural redução nas capacidades físicas e cognitivas, emergem dificuldades em relação ao cuidado dos longevos no ambiente familiar. Esse contexto obrigatoriamente desperta preocupações e incertezas com relação ao BES, considerado um componente essencial para a adaptação na vida tardia (Diener \& Chan, 2011), aliado às vivências afetivas e cognitivas, passíveis de análise pelo Teste de Pfister.

Diante do exposto, o tema da institucionalização também se apresenta como desafio necessário a ser investigado em seus benefícios e custos, tanto do ponto de vista social quanto em termos das características apresentadas pelos indivíduos envolvidos nesses processos. Nesse complexo contexto, o presente trabalho tem por objetivo investigar o BES de idosos longevos institucionalizados e residentes na comunidade, enfocando indicadores cognitivos e afetivos, por meio do TPC, além de componentes relativos à vivência das emoções e à autoestima. A partir de evidências empíricas obtidas com idosos em estudos prévios (Bastos-Formighieri \& Pasian, 2012; Carmo et al., 2012; Dutra \& Silva, 2014; Noronha et al., 2015; Oliveira et al., 2001), considerou-se plausível postular a hipótese de que idosos longevos residentes em ILPI apresentariam sinais de funcionamento cognitivo e socioafetivo diferenciado de residentes na comunidade, com hipótese de alguma disfunção nesses componentes psíquicos dos moradores em ILPI. Desse modo, esperar-se-ia sinais de prejuízo no funcionamento cognitivo e afetivo de idosos institucionalizados, com indicadores de depressão e de menor autoestima, comparativamente aos idosos não institucionalizados.

\section{Método}

\section{Participantes}

Participaram do estudo 70 idosos, com média etária de 83 anos $(D P=4,0)$, sendo $55(78,6 \%)$ do sexo feminino e 15 (21,4\%) do sexo masculino, voluntários do Estado do Rio Grande do Sul (RS). Os participantes, selecionados de forma não aleatória, foram distribuídos em dois grupos: institucionalizados $(\mathrm{GI}, n=35)$ e não institucionalizados (GNI, $n=35$ ). O GI foi procedente de 11 ILPIs privadas, três filantrópicas $(n=13)$ e oito com fins lucrativos $(n=22)$. O GNI foi proveniente de oito grupos de convivência social e da comunidade.

Os critérios de inclusão para os dois grupos de idosos foram: possuírem 80 anos de idade e mais, associado à ausência de: (a) histórico de situações estressoras decorrentes do diagnóstico de doenças graves ou de incapacidade física nos últimos seis meses; (b) histórico de perda que acarretou sofrimento psíquico significativo, com diagnóstico de depressão nos últimos seis meses; (c) comprometimento auditivo, visual ou motor que interferisse na execução das atividades avaliativas propostas; (d) déficit cognitivo. Os idosos de ILPI (grupo GI) deveriam residir na instituição há, no mínimo, seis meses.

\section{Instrumentos}

Questionário Sociodemográfico e de Saúde. Foi utilizado com o objetivo de verificar os critérios de inclusão da amostra. Elaborado pelas pesquisadoras, composto por 19 questões fechadas, relacionadas à idade, ao sexo, à situação conjugal, à escolaridade, ao nível socioeconômico e às condições de saúde.

Mini-Exame do Estado Mental (MEEM) (Folstein, Folstein, \& Mchugh, 1975). Traduzido e validado no Brasil por Bertolucci, Brucki, Campacci e Juliano (1994), objetivou avaliar aspectos cognitivos e selecionar os participantes. Composto por questões agrupadas em sete categorias, com escore total de zero a 30 pontos. Foram utilizados os pontos de corte do estudo de Brucki, Nitrini, Caramelli, Bertolucci e Okamoto (2003), ou seja, foram inclusos os voluntários com desempenho mínimo de: 21 pontos (um a quatro anos de escolaridade), 24 pontos (quatro a oito anos de escolaridade), 26 pontos (oito anos de escolaridade e mais). $\mathrm{O}$ instrumento apresentou, em estudo no Brasil, alfa de Cronbach de 0,80, evidenciando boa confiabilidade para aplicação em idosos (Santos, Cerchiari, Alvarenga, Faccenda, \& Oliveira, 2010).

Teste das Pirâmides Coloridas de Pfister (TPC) (Villemor-Amaral, 2013). Destinado à avaliação da personalidade, constituído por três esquemas de pirâmides e quadrículos de 10 cores, subdivididas em 24 tonalidades. $\mathrm{O}$ instrumento apresenta adequadas evidências psicométricas no contexto do Brasil, como já apontado. Neste estudo, foram analisadas as variáveis relacionadas ao aspecto formal e à frequência das cores das pirâmides, indicadores relacionados ao funcionamento cognitivo e afetivo, respectivamente.

Escala de Afetos Positivos e Afetos Negativos (PANAS) (Zanon et al., 2013). Oferece indicador 
relativo às vivências de afetos positivos e afetos negativos. Está constituída por 20 itens, em um formato do tipo Likert (1-5), sendo que o indivíduo deve escolher um número que represente quanto sente as emoções descritas pelos adjetivos. Quanto menor o número, menos intenso o afeto vivenciado, sendo o resultado expresso pela somatória dos pontos obtidos nos afetos positivos e nos afetos negativos. O estudo de validação da escala PANAS realizado com universitários (Zanon et al., 2013) apresentou alfa de Cronbach de 0,83 para afeto positivo e 0,77 para afeto negativo, considerados altos indicadores de sua precisão.

Escala de Depressão Geriátrica (GDS-15) (Sheikh \& Yesavage, 1986). Adaptada ao Brasil por Almeida e Almeida (1999), é considerada instrumento de rastreio de sintomas depressivos em idosos, contendo 15 perguntas com respostas dicotômicas (sim ou não), a respeito do humor da pessoa na última semana. O ponto de corte adotado, no valor maior ou igual a seis, corresponde a índices adequados de sensibilidade $(85,4 \%)$ e especificidade $(73,9 \%)$ para rastreio de sintomas depressivos. A consistência interna da escala apresentou alfa de Cronbach de 0,81, considerada alta (Almeida \& Almeida, 1999).

Escala de Autoestima de Rosenberg (EAR) (Rosenberg, 1965). Adaptada por Hutz e Zanon (2011), avalia a autoestima global por meio de 10 questões fechadas, direcionadas aos sentimentos de autoestima e autoaceitação. As questões organizadas em escala do tipo Likert (1-4 pontos) indicam que, quanto maior a pontuação, maior o nível de autoestima. A avaliação é unifatorial, em consonância com os achados de Rosenberg. A consistência interna da escala apresentou alfa de Cronbach de 0,90 , considerado valor bastante satisfatório (Hutz \& Zanon, 2011).

\section{Procedimentos}

O estudo recebeu aprovação do Comitê de Ética em Pesquisa da Universidade de Passo Fundo (RS), sob o parecer de número 886.141. Mediante a autorização das instituições, iniciou-se a coleta de dados no período de janeiro a agosto de 2015. Os idosos longevos assinaram o Termo de Consentimento Livre e Esclarecido (TCLE), responderam ao questionário sociodemográfico e ao MEEM. Após a triagem, os voluntários considerados elegíveis para o estudo realizaram o TPC, as escalas PANAS, GDS-15 e EAR, de modo individual e assistido, em uma hora, nas dependências de suas respectivas ILPIs ou grupos de convivência social. Os instrumentos foram aplicados e avaliados conforme seus respectivos padrões técnicos.

Os dados individuais foram cadastrados em planilha específica, recorrendo-se ao software IBM SPSS Statistics (versão 22.0), analisando-os de forma descritiva e comparativa, vistos os objetivos do estudo voltados à análise dos indicadores de vivências cognitivas e afetivas de idosos institucionalizados (GI) e não institucionalizados (GNI). Foram realizados testes de normalidade da distribuição dos resultados (teste de Kolmogorov-Smirnov), identificando-se que apenas a escala PANAS cumpriu esse pressuposto.

Para as análises estatísticas de comparação de resultados médios dos grupos de idosos, recorreu-se ao teste $t$ de Student na escala PANAS e, nos demais instrumentos, foi aplicado o Teste de Mann-Whitney ou Teste Exato de Fisher, sempre utilizando o nível de significância de 5\%. Foi calculado o $d$ de Cohen para obter o tamanho do efeito das diferenças identificadas nas análises comparativas, considerando-se: $d$ próximo a $0,20=$ efeito pequeno ou fraco, $d$ próximo a $0,50=$ efeito médio ou moderado, $\mathrm{d}$ maior que $0,80=$ efeito grande (Cohen, 1988). No caso de variável categórica (aspecto formal das pirâmides do TPC), foi utilizado o teste do qui-quadrado para comparar as frequências e proporções entre o GI e GNI.

\section{Resultados}

Diante dos objetivos propostos, os resultados iniciais estão relacionados à caracterização sociodemográfica e clínica da amostra alcançada. Esses resultados compõem a Tabela 1, sendo considerados o total dos participantes e a comparação entre GI e GNI.

Não foram identificadas diferenças estatisticamente significativas quanto às características sociodemográficas e clínicas dos participantes deste estudo. Os grupos não se diferenciaram em termos de condições de locomoção, embora no GI exista menor número de idosos que caminhe sem ajuda $(68,6 \%)$ em comparação ao GNI (82,9\%). A autopercepção de saúde positiva foi similar, concentrando-se como boa e regular nos dois grupos. Esses achados possibilitaram a avaliação comparativa dos idosos em termos das demais variáveis em foco neste trabalho, ou seja, seu funcionamento cognitivo e afetivo.

Considerou-se adequado apresentar a síntese dos resultados relativos aos instrumentos de avaliação psicológica utilizados em uma única tabela, de modo a facilitar sua análise integrativa. Desse modo, a Tabela 2 contém os resultados médios (e respectivo desvio padrão) das escolhas cromáticas e dos aspectos formais do Teste de Pfister (TPC), bem como os dados das escalas PANAS (afetos positivos e afetos negativos), GDS-15 (depressão) e EAR (autoestima) nos grupos, incluindo sua análise comparativa.

Com relação às variáveis examinadas do TPC neste trabalho, indicativas de componentes afetivos (escolhas cromáticas) e cognitivos (aspecto formal das pirâmides), não houve diferenças estatisticamente significativas entre GI e GNI. Pode-se, assim, sugerir preservação da dinâmica afetiva entre os idosos, assim como sinais de receptividade aos estímulos, independentemente de sua condição de moradia. 
Tabela 1

Características sociodemográficas e clínicas da amostra total e dos grupos

\begin{tabular}{|c|c|c|c|c|}
\hline \multirow[b]{2}{*}{ Variável } & \multicolumn{3}{|c|}{ Grupo (a) } & \multirow[b]{2}{*}{$p$} \\
\hline & $\begin{array}{c}\text { Total } \\
(n=70)\end{array}$ & $\begin{array}{c}\mathrm{GI} \\
(n=35)\end{array}$ & $\begin{array}{c}\text { GNI } \\
(n=35)\end{array}$ & \\
\hline Idade (anos) & $83(4,0)$ & $84(5,0)$ & $83(3,0)$ & $0,272^{\dagger}$ \\
\hline \multicolumn{5}{|l|}{ Sexo } \\
\hline Masculino & $15(21,4 \%)$ & $10(28,6 \%)$ & $5(14,3 \%)$ & \\
\hline Feminino & $55(78,6 \%)$ & $25(71,4 \%)$ & $30(85,7 \%)$ & $0,244^{*}$ \\
\hline \multicolumn{5}{|l|}{ Situação conjugal } \\
\hline Solteiro(a) & $13(18,6 \%)$ & $9(25,7 \%)$ & $4(11,4 \%)$ & \\
\hline Casado(a) & $9(12,9 \%)$ & - & $9(25,7 \%)$ & \\
\hline Viúvo(a) & $43(61,4 \%)$ & $22(62,9 \%)$ & $21(60,0 \%)$ & \\
\hline Separado(a) / divorciado(a) & $5(7,1 \%)$ & $4(11,4 \%)$ & $1(2,9 \%)$ & $0,005^{\dagger+1}$ \\
\hline \multicolumn{5}{|l|}{ Escolaridade } \\
\hline 0 a 4 anos & $52(74,3 \%)$ & $27(77,1 \%)$ & $25(71,4 \%)$ & \\
\hline 5 a 8 anos & $10(14,3 \%)$ & $3(8,6 \%)$ & $7(20,0 \%)$ & \\
\hline 9 a 11 anos & $4(5,7 \%)$ & $3(8,6 \%)$ & $1(2,9 \%)$ & \\
\hline 12 ou mais anos & $3(4,3 \%)$ & $2(5,7 \%)$ & $1(2,9 \%)$ & \\
\hline Não sabe & $1(1,4 \%)$ & - & $1(2,9 \%)$ & $0,392^{+1}$ \\
\hline \multicolumn{5}{|l|}{ Renda } \\
\hline Até um salário mínimo & $49(70,0 \%)$ & $27(77,1 \%)$ & $22(62,9 \%)$ & \\
\hline De 1 a 3 salários mínimos & $12(17,1 \%)$ & $4(11,4 \%)$ & $8(22,9 \%)$ & \\
\hline De 3 a 5 salários mínimos & $4(5,7 \%)$ & $2(5,7 \%)$ & $2(5,7 \%)$ & \\
\hline De 5 a 8 salários mínimos & $3(4,3 \%)$ & $1(2,9 \%)$ & $2(5,7 \%)$ & \\
\hline Não sabe & $2(2,9 \%)$ & $1(2,9 \%)$ & $1(2,9 \%)$ & $0,703^{+1}$ \\
\hline \multicolumn{5}{|l|}{ Filhos } \\
\hline Sim & $57(81,4 \%)$ & $26(74,3 \%)$ & $31(88,6 \%)$ & \\
\hline Não & $13(18,6 \%)$ & $9(25,7 \%)$ & $4(11,4 \%)$ & $0,218^{*}$ \\
\hline \multicolumn{5}{|l|}{ Caminha sozinho } \\
\hline Sim & $53(75,7 \%)$ & $24(68,6 \%)$ & $29(82,9 \%)$ & \\
\hline Não & $17(24,3 \%)$ & $11(31,4 \%)$ & $6(17,1 \%)$ & $0,265^{*}$ \\
\hline \multicolumn{5}{|l|}{ Estado de saúde } \\
\hline Excelente & $8(11,4 \%)$ & $2(5,7 \%)$ & $6(17,1 \%)$ & \\
\hline Bom & $44(62,9 \%)$ & $26(74,3 \%)$ & $18(51,4 \%)$ & \\
\hline Regular & $17(24,3 \%)$ & $7(20,0 \%)$ & $10(28,6 \%)$ & \\
\hline Muito ruim & $1(1,4 \%)$ & - & $1(2,9 \%)$ & $0,173^{+1}$ \\
\hline
\end{tabular}

Nota. (a) GI=grupo institucionalizado; GNI=grupo não institucionalizado; †Teste t de Student para amostras independentes (comparação de médias entre os dois grupos); ${ }^{\dagger+}$ Qui-quadrado (comparação entre os dois grupos); ${ }^{*}$ Teste Exato de Fischer (comparação entre os dois grupos)

Apesar da similaridade dos resultados dos idosos no TPC, cabe atenção especial a alguns indicadores. Nesse sentido, em termos descritivos, notou-se que as cores mais utilizadas foram o vermelho (vm1), azul (Az2) e o verde (vd2 e vd3), componentes da síndrome normal do TPC, sugerindo ajuste dos idosos ao esperado no instrumento, visto que são as cores com maior número de estímulos disponíveis. Por sua vez, as cores menos empregadas foram o branco, cinza e preto, que não possuem variação de tonalidades, novamente replicando a expectativa oferecida pelos estímulos apresentados. 
Tabela 2

Comparação dos Escores do TPC e das Escalas PANAS, GDS-15 e EAR nos Grupos

\begin{tabular}{|c|c|c|c|c|c|}
\hline & & Gru & & & \\
\hline & Variáveis & GI $(n=35)$ & GNI $(n=35)$ & & \\
\hline & & Média (DP) & Média (DP) & $d$ & $p$ \\
\hline Escolhas & Azul (Az) & $14,21(5,28)$ & $16,12(9,83)$ & 0,24 & $0,543^{* *}$ \\
\hline cromáticas & Vermelho (Vm) & $19,35(7,21)$ & $20,95(10,82)$ & 0,17 & $0,470^{*}$ \\
\hline & Verde (Vd) & $19,04(7,77)$ & $20,56(9,63)$ & 0,17 & $0,786^{* *}$ \\
\hline & Violeta (Vi) & $10,21(5,78)$ & $10,34(6,06)$ & 0,02 & $0,928^{*}$ \\
\hline & Laranja (la) & $11,74(8,57)$ & $8,75(6,13)$ & $-0,40$ & $0,140^{* *}$ \\
\hline & Amarelo (Am) & $10,34(5,62)$ & $9,45(7,62)$ & $-0,13$ & $0,271^{* *}$ \\
\hline & Marrom (Ma) & $5,19(4,38)$ & $4,75(4,29)$ & $-0,10$ & $0,694^{* *}$ \\
\hline & Preto (Pr) & $3,35(2,55)$ & $2,40(2,48)$ & $-0,38$ & $0,103^{* *}$ \\
\hline & Branco (Br) & $4,62(4,22)$ & $4,31(4,23)$ & $-0,07$ & $0,705^{* *}$ \\
\hline & Cinza (Ci) & $1,83(2,44)$ & $2,28(3,25)$ & 0,16 & $0,883^{* *}$ \\
\hline Aspectos formais & Tapete puro & 15 (42,9\%) & $16(45,7 \%)$ & 0,02 & $1,000^{\dagger}$ \\
\hline no TPC & Tapete furado & $24(68,6 \%)$ & $23(65,7 \%)$ & $-0,01$ & $1,000^{\dagger}$ \\
\hline & Tapete início de ordem & $9(25,7 \%)$ & $11(31,4 \%)$ & 0,07 & $0,791^{\dagger}$ \\
\hline & F. C. monotonais & $2(5,7 \%)$ & $4(11,4 \%)$ & 0,22 & $0,669^{\dagger}$ \\
\hline & F. C. monocromáticas & - & $2(5,7 \%)$ & 0,50 & $0,473^{\dagger}$ \\
\hline & F. C. multicromáticas & $29(82,9 \%)$ & $27(77,1 \%)$ & $-0,02$ & $0,765^{\dagger}$ \\
\hline & Formação simétrica & $1(2,9 \%)$ & $3(8,6 \%)$ & 0,31 & $0,607^{\dagger}$ \\
\hline & E. assimétrica dinâmica & $4(11,4 \%)$ & - & $-0,50$ & $0,122^{\dagger}$ \\
\hline Panas & Afetos positivos & $31,57(23,75)$ & $60,57(28,15)$ & 1,11 & $0,001^{* *}$ \\
\hline & Afetos negativos & $31,43(26,88)$ & $40,86(27,53)$ & 0,35 & $0,127^{* *}$ \\
\hline Depressão & GDS-15 & $8,11(1,58)$ & $7,40(1,41)$ & $-0,47$ & $0,071^{* *}$ \\
\hline Autoestima & EAR & $39,29(17,82)$ & $50,71(27,12)$ & 0,50 & $0,087^{* *}$ \\
\hline
\end{tabular}

Nota. (a) GI=grupo institucionalizado; GNI = grupo não institucionalizado; (b) FC=formação em camadas; E.=estruturas. Esses dados correspondem à frequência e porcentagem das variáveis em função do número de participantes dos grupos; *Teste $t$ de Student; ${ }^{* *}$ Teste de Mann-Whitney; †Qui-quadrado

Quanto ao aspecto formal, independentemente do contexto, os longevos exibiram maior quantidade de arranjos do tipo tapetes e reduzida ocorrência de formações e estruturas. Isso faz pensar na existência de um nível de funcionamento cognitivo menos elaborado, valendo-se mais das emoções e dos sentimentos, com sinais de reduzido funcionamento pautado em abstrações (condizente com estruturas e formações mais complexas).

No tocante aos indicadores da Escala PANAS, foi possível identificar diferença estatisticamente significativa (com grande tamanho de efeito) entre os dois grupos no total de afetos positivos. Os idosos de GI apresentaram menor escore de afetos positivos, sugerindo vivenciar menos sentimentos de entusiasmo, alegria e determinação, quando comparados ao GNI. O GI apresentou, ainda, menores escores de afeto negativo, quando comparados ao GNI, no entanto, sem diferença estatisticamente significativa. Pode-se supor a presença de sentimentos de medo, raiva, culpa, solidão, desprazer, tristeza e desânimo nos indivíduos avaliados, independentemente de seu contexto de residência, de acordo com os achados da PANAS.

Em relação ao rastreamento de depressão nos idosos avaliados, conforme resultados da GDS-15, não se detectou diferença estatisticamente significativa entre os grupos. Em GI e em GNI, o resultado médio atingiu pontuação superior à nota de corte (de seis pontos), sugerindo vivência de sintomas depressivos nos idosos, independentemente de seu local de moradia. O dado médio da GDS-15 foi superior em GI, com tendência à significância estatística na comparação com GNI, apontando maior intensidade de sinais depressivos entre os idosos institucionalizados.

Quanto à autoestima, inexistiram diferenças estatisticamente significativas nos resultados da EAR dos dois grupos. No entanto, essa comparação novamente tende à significância estatística, sendo que os idosos de GI apresentaram menores escores médios na EAR, quando 
comparado ao GNI. Os achados sugeriram sinais de menor satisfação consigo próprios e com a vida nos idosos em ILPIs, o que pode ser considerado consistente com os dados obtidos na PANAS (afetos positivos menores em GI).

\section{Discussão}

Ao avaliar o BES de longevos residentes em ILPIs e na comunidade, este estudo trouxe contribuições interessantes, tanto em relação aos dados sociodemográficos e clínicos, quanto relacionados aos instrumentos de avaliação psicológica. Foi possível notar menor autonomia funcional dos idosos do GI em relação ao GNI. Esse resultado apoia estudos sinalizadores de que, se por um lado o idoso residente em ILPI recebe cuidado e apoio, por outro lado a institucionalização pode incrementar perdas ao nível da autonomia e da privacidade (Carmo et al., 2012; Dutra \& Silva, 2014; Hammarstrom \& Torres, 2012; Marinho et al., 2013). Esse indicativo descritivo do nível de funcionalidade e de dependência de outros pode ter contribuído para os indicadores de decréscimo no BES entre os idosos institucionalizados (GI).

A literatura científica mostra que o comprometimento da capacidade funcional do idoso acarreta implicações importantes na execução das atividades da vida diária (Dutra \& Silva, 2014), incrementa a vulnerabilidade e a dependência na velhice, além de diminuir o BES e a qualidade de vida (Diener \& Chan, 2011). Alguns estudos sobre o BES menciona que os níveis de afeto negativo podem estar associados à falta de autonomia e à privacidade dos idosos (Hammarstrom \& Torres, 2012; Marinho, 2013).

Com relação aos aspectos afetivos e cognitivos obtidos a partir das variáveis examinadas do TPC, os idosos do GI e GNI apresentaram resultados semelhantes, sem diferenças estatisticamente significativas entre os grupos. Os achados foram sugestivos de nível de funcionamento cognitivo menos elaborado (predomínio de tapetes nas construções das pirâmides), mas acompanhado de sinais de manutenção da vivacidade emocional no conjunto de idosos (escolhas cromáticas similares ao esperado para sua faixa etária). Esses achados acompanham, em termos gerais, estudos que identificaram sinais de preservação da sensibilidade afetiva entre idosos (Bastos-Formighieri \& Pasian, 2012; Oliveira et al., 2001).

Esses resultados do TPC contrariam a hipótese inicial deste estudo, onde os idosos moradores em ILPIs teriam indicadores de funcionamento cognitivo e afetivo diferenciados dos idosos não institucionalizados. A condição de moradia, nesse sentido, não pareceu influenciar as variáveis das escolhas cromáticas e dos aspectos formais das pirâmides construídas pelos idosos de ambos os grupos.

As semelhanças entre os grupos podem estar relacionadas ao fato de a maioria dos participantes avaliados ser do sexo feminino, com média de idade semelhante. Ao pensar sobre essa característica sociodemográfica da atual amostra e suas escolhas cromáticas, pode-se destacar as cores mais frequentemente utilizadas e seu sentido interpretativo geral, como proposto no manual do TPC (Villemor-Amaral, 2013). Nesse sentido, vale a pena recorrer ao estudo de Villemor-Amaral et al. (2015), onde compararam a frequência das cores utilizadas entre mulheres/homens e meninas/meninos. Verificaram o uso de tonalidades mais enfraquecidas no grupo de mulheres (az2; vm1) e de meninas (az1; vm1). As autoras referiram que a preferência por tons mais claros pode estar culturalmente associada à feminilidade. Sendo assim, a escolha das cores pode sofrer a interferência de estereótipos culturais, o que também deve ser considerado ao se pensar nas escolhas cromáticas de idosos, embora ultrapasse os achados apresentados neste trabalho. Nesse momento, serão destacados apenas alguns elementos relativos às cores mais frequentemente utilizadas pelos idosos, a saber: vermelho, azul e verde, componentes da síndrome normal do TPC.

A cor mais utilizada pelos idosos foi o vermelho (vm), dado também encontrado no estudo de OliveiraCardoso e Santos (2014). O uso dessa cor, juntamente com a presença da tonalidade mais enfraquecida (vm1) e o azul em tonalidade leve (az2) pode contribuir para a desregulação dos mecanismos de controle dos afetos e impulsos (Villemor-Amaral, 2013). Tanto no GI quanto no GNI, as cores mais utilizadas foram o vermelho, o verde e o azul, o que corrobora os dois estudos prévios do TPC com idosos realizados no Brasil (BastosFormighieri \& Pasian, 2012; Oliveira et al., 2001). Embora esses resultados não confirmem a hipótese inicial deste estudo, nota-se a riqueza das informações alcançadas por meio do TPC, evidenciando preservação da adaptação social de idosos longevos. O TPC mostra-se, desse modo, instrumento de avaliação psicológica altamente promissor para a compreensão do funcionamento cognitivo e afetivo em idosos, embora ainda pouco explorado na literatura científica.

A cor azul apresentou-se predominantemente em tonalidade leve (az2), tanto em GI quanto em GNI. Segundo Villemor-Amaral (2013), a presença do azul deve estar marcada por tonalidades mais fortes (az4) para representar adequado controle e adaptação racional. Porém, a tonalidade que predominou entre os grupos de longevos deste estudo foi mais tênue (az2), podendo representar parciais mecanismos racionais de controle e de introversão. Essa possibilidade interpretativa ganhou força perante a elevada frequência dos tapetes nos aspectos formais das pirâmides, sinalizando elaboração racional pouco aprimorada entre os idosos. Nessa direção, os achados acompanharam, em parte, os dados de enfraquecimento no controle da impulsividade entre os idosos, apontado por Oliveira et al. (2001). 
Com relação à cor verde, GI apresentou predominância para a tonalidade vd2, enquanto em GNI houve predomínio da tonalidade vd3. A cor verde no TPC é representante do insight e da empatia, indica a capacidade da pessoa em se colocar no lugar do outro, de compreender cognitiva e emocionalmente os outros (Franco \& Villemor-Amaral, 2012; Nogueira, 2013). De acordo com Villemor-Amaral (2013), a cor verde está relacionada com boa capacidade de relacionamento e bom contato com o ambiente. Os resultados atuais confirmaram sinais de empatia e estabelecimento de relacionamentos afetivos e sociais entre os idosos avaliados. Esses achados legitimam estudos que encontraram boa qualidade de interação e adaptação socioafetivas em idosos (BastosFormighieri \& Pasian, 2012; Oliveira et al., 2001).

Com relação ao aspecto cognitivo (aspecto formal), GI e GNI apresentaram maior número de arranjos do tipo tapetes e diminuição de formações e estruturas. Isso sugere a presença de nível menos elaborado de funcionamento cognitivo entre os idosos dos dois grupos, valendo-se mais das emoções e sentimentos. Esses resultados de inexistência de diferenças estatisticamente significativas entre os grupos avaliados contraria a hipótese inicialmente formulada para este estudo.

Em termos gerais, poder-se-ia comentar que as evidências dos aspectos formais das pirâmides construídas por GI e GNI não pareceram influenciadas pela condição de moradia desses idosos, mostrando-se pouco elaboradas. A semelhança de proporção dos tapetes encontrados em GI e em GNI pode estar relacionada com o baixo nível de escolaridade dos idosos, o que mereceria exploração específica em futura análise, ultrapassando os objetivos propostos para este trabalho.

Cumpre destacar que a formação em tapetes foi encontrada em estudos com idosos (Oliveira et al., 2001) e com adolescentes e adultos, constatando-se associação com sinais de fragilidade estrutural, níveis não satisfatórios de amadurecimento, emoções e manejos defensivos, além de indicadores de desorganização cognitiva e emocional (Nogueira, 2013; Villemor-Amaral et al., 2005). Alguns fatores como a incapacidade funcional, a falta de autonomia e a idade avançada, quando presentes no contexto da institucionalização, podem incidir no funcionamento cognitivo do idoso acentuando sua vulnerabilidade e dependência, o que pode contribuir negativamente na qualidade de vida e no BES (Diener \& Chan, 2011; Dutra \& Silva, 2014; Oliveira et al., 2012).

Em complemento aos dados do TPC, foi possível colecionar evidências relativas às vivências dos afetos dos idosos por meio do PANAS. Os resultados médios dos afetos positivos nessa escala foram estatisticamente diferenciados entre GI e GNI, evidenciando menos sentimentos de bem-estar, como alegria, amabilidade, determinação, entusiasmo em idosos institucionalizados.

Nos afetos negativos não foi identificada diferença estatisticamente significativa entre os grupos avaliados, embora GI tenha média inferior, sugerindo menos sentimentos de medo, de raiva, de culpa, de solidão, de nervosismo nesse grupo, quando comparado ao GNI. No entanto, há que se levar em consideração nessa análise que os idosos institucionalizados apresentaram menores resultados de expressão emocional no PANAS (na subescala Afetos positivos e na subescala Afetos negativos), o que pode ser compreendido como sinal de inibição afetiva geral entre idosos institucionalizados.

A presença de sentimentos de afeto negativo, como angústia, desespero, insegurança, abandono, saudade (Carmo et al., 2012) podem ser considerados fatores de risco para o desenvolvimento de sintomas depressivos (Vicente, et al., 2014). Esses elementos, por sua vez, tendem a interferir na funcionalidade do invidíduo, podendo contribuir para a diminuição da qualidade de vida e bem-estar em idosos (Carmo et al., 2012; Dutra \& Silva, 2014).

Nessa direção, faz sentido explorar os achados relativos ao rastreamento de sinais depressivos, obtidos pela Escala GDS-15. Os resultados médios foram semelhantes entre os grupos, apontando indicadores de depressão em todos os idosos avaliados, independentemente de sua condição de moradia. A depressão é um sintoma comum em idosos, encontrando-se associada à percepção negativa da própria experiência de vida, a sentimentos negativos, como medo, raiva, solidão e aos afetos negativos (Noronha et al., 2015; Vicente et al., 2014). Os longevos do GI e do GNI também evidenciaram sentimentos negativos (medo, raiva, culpa solidão, tristeza e nervosismo) na escala PANAS, porém sem diferenças estatisticamente significativas entre os grupos.

Estudos realizados com idosos institucionalizados destacaram os sentimentos negativos, principalmente a solidão, como fatores motivadores de sintomas depressivos em idosos (Carmo et al., 2012; Marinho et al., 2013; Vicente et al., 2014). Os referidos estudos apontaram a depressão como um transtorno comum entre idosos, principalmente os institucionalizados, o que pode contribuir para a emergência de sentimentos negativos vivenciados pela institucionalização. Depreende-se, portanto, que a vivência de sentimentos depressivos nos idosos pode contribuir para a percepção depreciativa sobre a própria institucionalização e no baixo afeto positivo (Carmo et al., 2012).

Por fim, esses resultados e considerações interpretativas devem ser vinculados aos dados relativos à autoestima derivados da escala EAR. Os idosos não apresentaram diferenças estatisticamente significativas no escore médio dessa escala, porém GI sinalizou menores escores de autoestima quando comparados aos idosos do GNI, além de rebaixamento do afeto positivo na PANAS. Esses resultados validam a hipótese inicial deste estudo, ou seja, baixos escores de autoestima e vivências afetivas positivas menos vívidas em idosos institucionalizados, 
diferenciando-se da afetividade de idosos residentes em seus lares. Outros estudos com idosos referem baixos escores de satisfação com a vida, presença de afetos negativos e rebaixamento de afeto positivo (Noronha et al., 2015; Oliveira et al., 2012; Tinoco et al., 2015).

Os atuais resultados de rebaixamento dos escores de afeto positivo na PANAS em GI podem ser compreendidos como relacionados a estudos que referem sentimentos negativos (como tristeza, raiva e solidão) contribuindo para o rebaixamento do afeto positivo e a emergência de sintomas depressivos (Noronha et al., 2015; Oliveira et al., 2012; Vicente et al., 2014; Zanon et al., 2013). Podese presumir que os idosos do GI vivenciaram emoções positivas durante a vida, porém com menos intensidade do que o GNI, o que precisaria ser melhor investigado. A presença de sentimentos de afetos negativos e o rebaixamento da autoestima, também pode levar a pessoa a sentir-se mais triste, desestabilizando-se emocionalmente (Noronha et al., 2015; Oliveira et al., 2012).

Outra possibilidade de compreensão desses achados vincula-se ao modo como a institucionalização é vivida pelos idosos, podendo significar abrigo e proteção (Carmo et al., 2012), ao mesmo tempo em que representa perda do convívio familiar e rompimento com vínculos afetivos, podendo reduzir a alegria e o entusiasmo. Pontuações baixas de afeto positivo foram constatadas em indivíduos que vivem episódios de desprazer intensos e frequentes (Noronha et al., 2015; Oliveira et al., 2012; Zanon et al., 2013). Observou-se, neste estudo, que os sentimentos depressivos e negativos em idosos do GI foram mais proeminentes, quando comparados aos afetos positivos.

Os afetos positivos evoluem ao longo da vida, com a maturidade, vinculados ao aumento da capacidade de regulação das emoções (Zanon et al., 2013). Há evidências de que experimentar emoções positivas pode não só melhorar a qualidade de vida, como acrescentar mais anos à vida (Diener \& Chan, 2011).

Os idosos do GI apresentaram sinais de inibição emocional, com menor presença de afetos positivos quando comparados aos GNI. Esse dado apoia estudos que destacam a capacidade de lidar com as emoções como essencial para alcançar o BES (Hutz et al.; 2014; Zanon et al., 2013). Ou seja, quanto mais os indivíduos acreditam em si mesmos, maior é o senso de merecimento de eventos positivos, o que pode aumentar o BES. Altos escores de satisfação de vida estão associados a elevados níveis de afetos positivos e baixos níveis de afetos negativos (Zanon et al., 2013).

Por fim, muito embora este estudo tenha trazido resultados interessantes, eles devem ser interpretados sob a luz de algumas limitações. Eles advêm de amostra não probabilística e de um grupo restrito de idosos residentes em ILPIs privadas e frequentadores de grupo de convivência social de um contexto específico, predominantemente mulheres, o que não permite generalização. Além disso, os resultados obtidos derivam de estudo descritivo-comparativo e não permitem inferir associações de causa-efeito ou associações entre as diferentes variáveis dos instrumentos de avaliação psicológica utilizados. No que diz respeito a sua aplicabilidade, os achados oferecem indícios para se pensar em ações promotoras de uma velhice balizada na autonomia e interação social dos longevos e, consequentemente, melhor qualidade de vida. Mediante os dados apresentados e a relevância da temática investigada com a população longeva emergente, sugere-se o seguimento de estudos com amostras representativas e com incremento da inclusão do sexo masculino, a fim de identificar os fatores que podem contribuir para a promoção do BES.

\section{Referências}

Almeida, O. P., \& Almeida, S. A. (1999). Confiabilidade da versão brasileira da escala de depressão em geriatria (GDS) - versão reduzida. Arquivos de Neuro-Psiquiatria, 57(2-B), 421-426. doi: 10.1590/S0004-282X1999000300013

Bastos-Formighieri, M. D. S., \& Pasian, S. R. (2012). O Teste de Pfister em Idosos [The Pfister Test in the elderly]. Avaliação Psicológica, 11(3), 435-448. Recuperado de http://pepsic.bvsalud.org/pdf/avp/v11n3/v11n3a10.pdf

Bertolucci, P. H. F., Brucki, S. M. D., Campacci, S. R., \& Juliano, Y. (1994). O mini-exame do Estado mental em população geral: Impacto da escolaridade. Arquivos de Neuropsiquiatria, 52(1), 1-7. doi: 10.1590/S0004-282X1994000100001

Brucki, S. M. D., Nitrini, R., Caramelli, P., Bertolucci, P. H. F., \& Okamoto, I. H. (2003). Sugestões para o uso do Mini-Exame do Estado Mental no Brasil. Arquivos de Neuropsiquiatria, 61(3-B), 777-781. doi:10.1590/S0004-282X2003000500014

Carmo, H. O., Rangel, J. R. A., Ribeiro, N. A. D. P., \& Araújo, C. L. D. O. (2012). Idoso institucionalizado: O que sente, percebe e deseja? Revista Brasileira de Ciências do Envelhecimento Humano, 9(3), 330-340. doi: 10.5335/rbceh.2012.046

Cohen, J. (1988). Statistical power analysis for the behavioral sciences (2 ${ }^{\mathrm{a}} \mathrm{ed}$.). Hillsdale: Lawrence Erlbaum Associates. Inc.

Diener, E., \& Chan, M. Y. (2011). Happy People Live Longer: Subjective Well-Being contributes to health and longevity. Applied Psychology: Health and Well-Being, 3(1), 1-43. doi: 10.1111/j.1758-0854.2010.01045.x

Dutra, F. C. M., \& Silva, H. R. O. (2014). Bem-estar subjetivo, funcionalidade e poio social em idosos da comunidade. Estudos Interdisciplinares do envelhecimento humano, 19(3), 775-791. Recuperado de https://seer.ufrgs.br/RevEnvelhecer/article/view/43389/33285

Folstein, M. F., Folstein, S. E., \& Mchugh, P. R. (1975). Mini-mental state: A practical method for grading the cognitive state off patients for the clinician. Journal of Psychiatric Research, 12(3), 189-198. doi: 10.1016/0022-3956(75)90026-6 
Franco, R. D. R. C., \& Villemor-Amaral, A. E. D. (2012). Validade incremental do Zulliger e do Pfister no contexto da toxicomania. Psico-USF, 17(1), 73-83. doi: 10.1590/S1413-82712012000100009

Hammarstrom, G., \& Torres,S. (2012).Variations in subjective well-being when, aging in place - A matter of acceptance predictability and control. Journal of Aging Studies, 26(2), 192-203. doi: 10.1016/j.jaging.2011.12.004

Hutz, C. S., \& Zanon, C. (2011). Revisão da adaptação, validação e normatização da escala de autoestima de Rosenberg. Avaliação Psicológica, 10(1), 41-49. Recuperado de http://pepsic.bvsalud.org/pdf/avp/v10n1/v10n1a05.pdf

Hutz, C. S., Midgett, A., Pacico, J. C., Bastianello, M. R., \& Zanon, C. (2014). The Relationship of Hope, Optimism, Personality in Brazilians and Americans. Psychology, 5(6), 514-522. doi: 10.4236/psych.2014.56061

Marinho, L. M., Vieira, M. A., Costa, S. M., \& Andrade, J. M. O. (2013). Grau de dependência de idosos residentes em instituições de longa permanência. Revista Gaúcha de Enfermagem, 34(1), 104-110. doi: 10.1590/S1983-14472013000100013

Nogueira, T. D. G. (2013). O teste de Pfister na avaliação de depressão e ansiedade em universitários: Evidências preliminares. Boletim de Psicologia, 63(138), 11-21. Recuperado de http://pepsic.bvsalud.org/pdf/bolpsi/v63n138/v63n138a03.pdf

Noronha, A. P. P., Martins, D. D. F., Campos, R. R. F., \& Mansão, C. S. M. (2015). Relações entre afetos positivos e negativos e os cinco fatores de personalidade. Estudos de Psicologia, 20(2), 92-101. doi:10.5935/1678-4669.20150011

Oliveira-Cardoso, É. A. D., \& Santos, M. A. D. (2014). Psicodinâmica dos transtornos alimentares: indicadores do Teste das Pirâmides Coloridas de Pfister. Psico USF, 19(2), 209-220. doi: 10.1590/1413-82712014019002006

Oliveira, É. A. D., Pasian, S. R., \& Jacquemin, A. (2001). A vivência afetiva em idosos. Psicologia: Ciência e Profissão, 21(1), 68-83. doi: 10.1590/ S1414-98932001000100008

Oliveira, S. F., Queiroz, M. I. N., \& Costa, M. L. A. (2012). Bem estar subjetivo na terceira idade. Motricidade, 8(S2), 1038-1047. Recuperado de http://www.redalyc.org/articulo.oa?id=273023568131

Pasian, S. R., Barroso, J. B., \& Theodozio, D. R. (2014). O Teste de Pfister na adolescência. In A. E. Villemor-Amaral (2014). As Pirâmides de Pfister com Crianças e Adolescentes (pp. 107-126). São Paulo: Casa do Psicólogo.

Rosenberg, M. (1965). Society and the adolescent self-image. Princeton: Princeton University Press.

Santos, C. S., Cerchiari, E. A. N., Alvarenga, M. R. M., Faccenda, O., \& Oliveira M. A. C. (2010). Avaliação da confiabilidade do mini-exame do estado mental em idosos e associação com variáveis sociodemográficas. Cogitare Enferm, 15(3), 406-412. doi: 10.5380/ ce.v15i3.18879

Sheikh, J. I., \& Yesavage, J. A. (1986). Geriatric Depression Scale (GDS): Recent evidence and development of a shorter version. Clinical Gerontology, 5(1-2), 165-173. doi: 10.1300/J018v05n01_09

Tinoco, D. O., Tinoco, B., Ackermann, L., \& Carvalho, A. L. N. (2015). Envelhecimento saudável: O que dizem os idosos e os profissionais da área de psicologia. Polêmica, 15(1), 47-60. doi: 10.12957/polemica.2015.16008

Vicente, F., Espirito-Santo, H., Cardoso, D., Silva, F. D., Costa, M., Mártins, S., ... \& Moitinho, S. (2014). Longitudinal study of factors associated with the development of depressive symptoms in institutionalized elderly. Jornal Brasileiro de Psiquiatria, 63(4), 308-316. doi: 10.1590/0047-2085000000039

Villemor-Amaral, A. E., Primi, R., Farah, F. H. Z., Silva, S. D., Cardoso, L. M., \& Franco, R. R. C. (2004). A depressão no teste das pirâmides coloridas de pfister. Paidéia (Ribeirão Preto), 14(28), 69-176. doi: 10.1590/S0103-863X2004000200006

Villemor-Amaral, A. E., Primi, R., Franco, R. R. C., Farah, F. H. Z., \& Cardoso, L. M. (2005). O Teste de pfister e sua contribuição para diagnóstico da esquizofrenia. Revista do Departamento de Psicologia, UFF, 17(2), 89-98. doi: 10.1590/S0104-80232005000200008

Villemor-Amaral, A. E., Biasi, F. C., Cardoso, L. M., Pavan, P. M. P., \& Tavella, R. R. (2015). Pink and Blue: Sex and Age in the Pfister's Test. Psico-USF, 20(3), 411-420. doi: 10.1590/1413-82712015200304

Villemor-Amaral, A. E. D., Tavella, R. R., Cardoso, L. M., Biasi, F. C., \& Pavan, P. M. P. (2014). Teste das pirâmides coloridas de Pfister e a criatividade em crianças. Psicologia: Teoria e Prática, 16(3), 114-124. doi: 10.15348/1980-6906/psicologia.v16n3p114-124

Villemor-Amaral, A. E., Farah, F. H. Z., \& Primi, R. (2004). O Teste das pirâmides coloridas e o Transtorno do Pânico. Psicologia em Estudo, 9(2), 301-307. doi: 10.1590/S1413-73722004000200016

Villemor-Amaral, A. E. (2013). As pirâmides coloridas de Pfister. (2nd ed.). São Paulo, SP: Casa do Psicólogo.

Zanon, C., Bastianello, M. R., Pacico, J. C., \& Hutz, C. S. (2013). Desenvolvimento e validação de uma escala de afetos positivos e negativos. Psico-USF, 18(2), 193-201. doi: 10.1590/S1413-82712013000200003

\section{Sobre as autoras}

Cristina Ribas Teixeira é psicóloga, mestra em Envelhecimento Humano pela Universidade de Passo Fundo (UPF). Atualmente, é professora no Curso de Psicologia do Instituto de Desenvolvimento Educacional de Passo Fundo.

Silvana Alba Scortegagna é psicóloga, doutora em Psicologia pela Universidade São Francisco (USF). Atualmente, é Professora no Programa de Pós-Graduação Stricto Sensu em Envelhecimento Humano da Universidade de Passo Fundo.

Sonia Regina Pasian é psicóloga, doutora em Ciências pela Universidade de São Paulo (USP). Atualmente, é professora no Programa de Pós-Graduação Stricto Sensu em Psicologia da Universidade de São Paulo, Ribeirão Preto.

Marilene Rodrigues Portella é enfermeira, doutora em Enfermagem pela Universidade Federal de Santa Catarina (UFSC). Atualmente, é Professora no Programa de Pós-Graduação Stricto Sensu em Envelhecimento Humano da Universidade de Passo Fundo. 